\title{
Prospects for Reducing Accidents on the Roads of the Russian Federation Using the Scientific and Methodological Approach
}

\author{
Sergey Glagolev ${ }^{1}$, Aleksander Novikov ${ }^{2}$, Ivan Novikov ${ }^{1}$, and Anastasia Shevtsova ${ }^{1 *}$ \\ ${ }^{1}$ Belgorod State Technological University named after V.G. Shukhov, 46 Kostyukova str., Belgorod, \\ 308012, Russia \\ ${ }^{2}$ Orel State University, 95 Komsomolskaya str., Orel, 302026, Russia
}

\begin{abstract}
This article reports the results of an in-depth analysis of the transport situation in the Russian Federation and presents the main programmes, aimed at its improvement. The paper deals with the major ways of reducing risk of traffic accidents and increasing traffic safety. The paper proposes the scientific and methodological approach, providing the specialists with the opportunities of conducting a comprehensive assessment of the causes of emergencies and aiming at eliminating road accidents rates. The origin of the process of an emergency situation occurrence has been investigated and designed, the key components (constituents) of the process have been identified and the basic mechanisms, influencing the basic components, have been analyzed. The algorithm of the application of the proposed and developed scientific and methodological approach at the level of departmental organizations has been suggested. The article describes the mathematical model of the process of emergency situations occurrence, which, together with the applied telematics systems and intelligent transport systems, will help to achieve the basic objectives of the study.
\end{abstract}

\section{Introduction}

The transport situation, prevailing in many regions of the country, resembles the tendency typical for many countries of the world, indicating that the high growth of motorization and number of cars on the roads, leads to increased growth of traffic accidents. In order to reduce the accident rate indicator and the number of road accidents the leaders of the country have elaborated, developed and adopted the programmes at the federal level, which will improve the current transport situation. So far, one of the main programmes for improving road safety, is the special federal programme under the title "Improvement of road, traffic safety in the period from 2013 to 2020» (approved by the Decree of the Government of the Russian Federation from October 3, 2013 N 864) [1].

The programme sets a target of achieving decline and fall of the number of trafficrelated deaths by 8,000 people $(28.82 \%)$ by 2020 , which is definitely less than in 2012 [1].

* Corresponding author: shevcova-anastasiya@mail.ru 
According to statistics, provided by the official Russian traffic police, 203,597 road accidents occurred in Russia in 2012, in which 27,991 people died and 258,618 were injured. [2]. According to data released by Rosstat (Head Federal State Statistics Service of the Russian Federation), the number of cars is constantly growing, increasing from 257,5 per 1000 inhabitants in 2012 to 288.8 in 2015 , and thus, this three-year period witnessed an increase by $12 \%$ [3] It is a complicated task to handle to achieve the main objective of the existing road safety programme with such figures and rates, but, according to data and indicators, it is being successfully implemented. Data analysis shows that we are observing a significant drop and lowering of these figures in the Russian Federation as a whole in the process of the programme implementation, which is the demonstration of its validity, efficiency and effectiveness, but let's consider the analyzed indicators in areas located in the Central Federal District (CFD) [4] in more detail (figure 1).

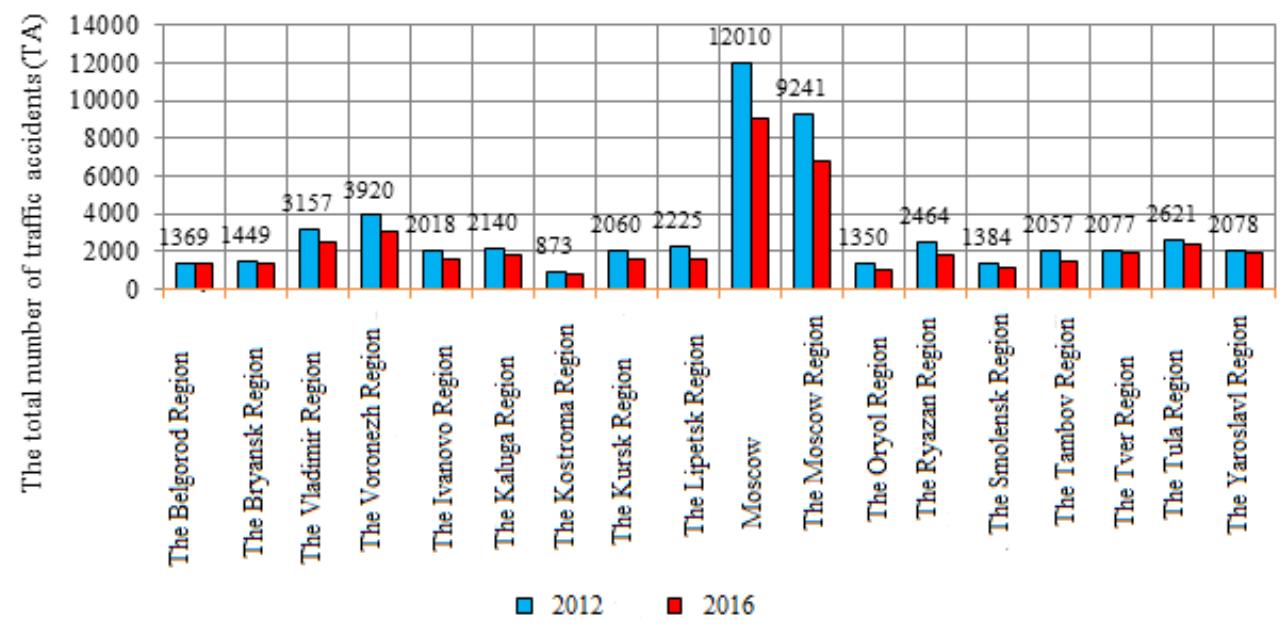

Fig. 1. The histogram of the number of traffic accidents in the CFD in the period from 2012 to 2016.

Obviously, there is a positive trend of reducing accidents not only in the CFD (which is in the focus of this paper), but also in Russia in general, and the purpose of the existing Federal Target Programme (FTP) as of 2016 is achievable and feasible, despite the fact that the deadline is 2020 .

\section{Development of the model, representing the situations, when emergency arises}

A holistic (complex) approach, embodied in the main purpose and objective of the existing Federal Target Programme, comprises such aspects as early upbringing, training and teaching people the so called driving culture, as well as using preventive activities and enhancing security in case an accident has already happened, which involves the measures, aimed at improving the system of providing the victims of the road accidents with assistance, thus, the tasks, mentioned above, can be divided into initial and final by analogy with the process of occurrence of an accident [5]. Particular attention in this context should be given to the initial objectives, they are being successfully implemented in the existing programme, but, unfortunately, they are of a preventive nature, namely building up the strategic approaches to forming the culture of driving before the occurrence of an emergency situation, because the majority of traffic accidents is the fault of the careless and negligent drivers $(86.8 \%$ according to the data released in 2016). It should be mentioned 
that, when the accident takes place, it is usually a consequence of the combination of a large number of factors and reasons, which affect its character and do not dependent on a driver's behavior and the culture of driving. The culture of driving itself will help the driver, who is an active participant of traffic, to perform the necessary actions, avoiding an emergency situation, but in most cases particular conditions inevitably precede an accident, and the driver himself can do almost nothing to prevent or to cope with them, therefore, there is an extremely important challenge in building scientific and methodological foundations of forecasting and preventing traffic accidents by means of applying the system-information approach. A thorough study of this field will help to develop some practical recommendations and specialized software, thus, reducingthe number of traffic accidents.

The research may be conducted from the scientific standpoint. A particular direction to the study may be given by science, which allows us to investigate and develop an information model of the traffic accident, using the system information toolkit, providing a comprehensive analysis of the components of the «driver-vehicle-road-medium» system [6]. An expended and advanced investigation of accident prediction and prevention is aimed at reducing the number of fatalities and injuries in various road accidents by making the total number of such accidents fall and come down.

According to the main objective, a new integrated approach to the study of the initial stage of an accident must be applied and requires implementation together with the investigation of the main factors, presented in Figure 2, and inherent in objects, influencing the occurrence of an emergency.



Fig. 2. The components of an emergency.

For a more careful and in-depth analysis you need a more detailed consideration of the constituents of the emergency situation, which can lead to a traffic accident. The combination of a large number of factors in the process of driving a car may cause the situations, undergoing our analysis; therefore, close and systematic description of these factors and development of the measures for their analysis will help to reduce the probability of their occurrence.

\section{An analysis of the constituents of the developed model}

According to the diagram (figure 2) a lot of constituents and components have a considerable impact upon the occurrence of emergency situations, which usually give rise to a great deal of traffic accidents, and can be roughly divided into the following groups:

1. A driver $(k d)$ is one of the most important components of the process of an accident. When considering the traditional and classic «driver-vehicle-road-medium» form of the traffic process it is fashionable to say, that without human («driver») intervention and 
participation this process cannot be implemented. Introducing and reflecting the process of emergency in the scheme, provided in this article (fig. 2), we must emphasize the fact, that the driver is also an integral part of it and plays an important role in this process. Developing an information model of traffic accident we should clearly formulate and understand all the components of the factors, contributing to its emergence, so, it's recommended to define a driver's $(k d)$ indicators and characteristics, such as: age [7], driving experience; psychological state; a type of temperament.

In the process of performing this scientific research we may achieve clear understanding of manner of the object («the driver») behaviour, which is of vital importance for the correct formation of an information model of the traffic accident. So, due to introducing the coefficient «the driver» $(k d)$ creation of its coded signs and indicators with the detailed deciphering of its values will be possible, for example, by means of using the system in the range from 0 to 1 , we will be able to classify the type of the driver and assess his impact upon the process of the emergency:

In case $k d=0$ the object «the driver» does not influence the model of the traffic accident. The driver, belonging to a certain age group, has not made any mistakes in this transport situation, his driving experience has been sufficient for assessing the situation in a proper way and making the right decision, his psychological state and condition have been satisfactory, he has been showing a high level of an ability to perform work and maximize time, and the temperament type, characteristic of this object, was not alarming and dangerous in the present transport situation.

In case $k d=1$ the "driver" has a significant (maximum) impact on the model of the traffic accident, it is important to take into consideration the remaining components, such as the place (location) of an accident, a vehicle, traffic flow, external conditions, but the basic factor of occurrence of the traffic accident depends on the object, i.e. «the driver». The driver, belonging to a certain age group, made all the possible, various and divergent errors in the given transport situation, has not committed the actions necessary for this situation, his driving experience has not been sufficient for assessing the situation and making the right decision, psychological state has not been satisfactory, his performance has been minimal, and the type of temperament, characteristic of this object was not alarming and dangerous in the transport situation.

Study of the intermediate values, characteristic for the $k_{d}$ coefficient «the driver» will help to build up a mathematical model of his behavior, which would be one of the main pillars in the development of an information model of the phenomenon of the traffic accident itself.

2. Traffic (transport) flow $(k t f)$ is a part and a constituent of emergency situations. It is one of the new fields and directions in the process of considering the phenomenon of the traffic accident, which has not been previously included into the research and focus of investigation of these types of accidents. According to the existing research, being conducted in the field of traffic management, there are two systems of the classifications of the state of transport (traffic) flow [8].

These kind of parameters (markers) under consideration are, obviously, interconnected and are characterized by such values of various parameters (markers) and indicators as speed, density and saturation (richness) (the level of the amount of the cars on the roads). The research work, extending Professor V.F. Babkov's researches, is undertaken at Tyumen State Oil and Gas University nowadays [8]. In his writings he classifies certain kinds of accidents, which are unique and characteristic for the specific traffic conditions and the researches, that prove the impact of existing levels of convenience on the types of accidents, have been conducted, so, a certain number of accidents is typical of each level of convenience. 
We must conduct a complex research of the object under consideration and form the classification system in the range from 0 to 1 , which have been accepted earlier.

If $k t f=0$, so, the object «traffic flow» does not influence the model of the road accident. Traffic conditions are characterized as free, cars in the traffic flow have no impact upon each other, the level of load is minimal, the flow velocity and speed does not violate the permissible limit on the specific part of the road under consideration, and richness and density of traffic flow are minimal.

If $k d=1$, so, the object «traffic flow» has a significant (maximum) impact on the model of traffic accident, it is necessary to take into account other constituents, such as a place of the accident, a vehicle, a driver, external conditions, but the main factor, causing the accident, has been found out in the process of investigation of the object «traffic flow». Traffic conditions are satisfactory, the cars are moving in rows with frequent stops, speed is very low, traffic richness (saturation)and density are enormous (maximum).

3. The vehicle $\left(k_{v}\right)$ is the third constituent of the situation of the road accident. By representing the process of vehicle movement in the classical «driver-vehicle-road-medium form, we have come to the conclusion, that the car is one of the most hazardous and dangerous constituents, the preservation of human life (reduction of fatality) depends directly on the level of its deformation. Investigation of this object should also be carried out in an integrated manner, i.e. with an analysis of the main indicators of its security, both active and passive [9].

If $k_{v}=0$, then the object «vehicle» does not influence the model of the road accident. The actions of the constituents of an active safety and security are maximum; we do not consider the object to be of a significantly hazardous or deleterious nature, thus, risk of the accident for this object is very low (minimal).

If $k_{v}=1$, so, the object «vehicle» has a great impactupon the model of the road accident, and other constituents, such as the place of the accident, the traffic and transport flow, the driver, the external conditions should also be taken into consideration, but the major factor of the accident has been figured out, when this object was undergoing our analysis. If one or more elements of the so called «active» security fail and break down, probability of the traffic accident is extremely high.

4. External conditions $\left(k_{e c}\right)$. This component of the traffic accident, being considered as a research object, allows us to assess all the parameters, which characterize the external conditions, existing and occurring during the movement of the car, quite comprehensively. According to the task of the research, it is necessary to define impact of the road conditions in the process of conducting the analysis of the parameters of the constituents, comprising the object «external conditions».

If $k_{e c}=0$, so, the object «external conditions» does not have the slightest impact upon the traffic accident model. Influence of the factors of road conditions is minimal and poses no hazard.

If $k_{e c}=1$, so, the object «external conditions» has a considerable (maximum) impact on the traffic accident model. Again it is vital to pay attention to and take into account such components as the place of the accident, traffic flow, the driver, the vehicle), but the main factor, causing the accident, has been found out in the process of investigation of the object. If one or more elements of the road conditions are risky and dangerous, so, probability of the traffic accident is extremely high.

5. The place of the accident is the last constituent of the phenomenon under our consideration. This constituent can not be treated in the framework of the emergency, because this object is referred to the moment, which usually takes place after the traffic accident, i.e. to the event (the phenomenon), which has already happened, but it should be analyzed precisely from the angle of this aspect, and such indicators as a hotbed of 
accidents [10] and the place of the concentration of the traffic accident should be considered [11].

The investigation of the hotbeds of accidents and the places of the concentration of the traffic accidents will help us to find out and define the part of the road, where the accident occurred. There we can apply the information model of the traffic accident, including and comprising all the objects (constituents) under consideration, which were described earlier (above), therefore, the mathematical model of the traffic accident, which requires further analysis, will be represented and written in the following way:

$$
T A=k d+k v+k e c+k t f \text {. }
$$

\section{Conclusion}

Implementation of the apparatus, represented in the paper, provides us with assistance in development of specialized software, which users are the representatives of an executive branch of power, who should make proper decisions, concerning the formulation of practical recommendations to reduce the number of accidents and achieve the main objective of the research, on the basis of data, integrated and united into the developed programme.

In the process of carrying out the research several up-to-date, acute and sophisticated transport problems can be solved due to the implementation and application of the apparatus of the traffic accident emergence and taking into consideration possible factors, influencing it in the process of investigation of the transport system and application of intellectual transport systems [12,13] with the built-in telematics devices [14,15]. Such a large-scale research, focusing on the complexity of integration and interaction of all the factors, has not been conducted yet in this field, that is why this direction of the research has a lot of positive aspects and prospects, so, its implementation is extremely important and essential, because it will help us to increase transport efficiency of the entire transport and road traffic complex of the country.

The article has been prepared in the framework of the programme of the foundation (basic) university development on the basis of BSTU named after V.G. Shuhov.

\section{References}

1. The Federal Target Programme «Strengthening and maximising traffic safety in the period from 2013 to 2020» (approved under Decree of the Government of the Russian Federation No 864, dated 03.10.2013).

2. The markers of the level of traffic security. Available at: http://www.gibdd.ru/stat/.

3. Statistics of the Russian Federation. Available at: http://www.gks.ru/.

4. The Central Federal District. Available at: http://cfo.gov.ru/.

5. A.V. Saraev, E.A. Novopisnyj, S.V. Dorohin, I.A. Novikov, Some common questions of examination and expertise of road accidents. Monograph. Belgorod (2015)

6. I.A. Novikov, A.E. Borovskoj, A.G. Shevcova, Cotrol and management of traffic in the process of a single element of "VADS" system. Information technologies and innovations on the field of transport, Materials of an international scientific and practical conference, ed. A.N. Novikov, pp. 231-238 (2015)

7. E.V. Kaz'mina, E.I. Zheleznov, Influence of the driver's age and driving experience on secure and safe manner of driving. Izvestija VolgSTU, «Land transportation resources», Volgograd, 5, no 2, pp. 64-66 (2012) 
8. V.F. Babkov, Road conditions and traffic safety. M.: Transport, 271 p. (1993)

9. N.Ja. Jah'jaev, Safety of transport means (vehicles). M.: Academy Publishing, 432p. (2011)

10. Methodological manual on the training of specialists on road safety in road transport (approved by the Ministry of Transport of the Russian Federation).

11. ODM 218.6.015-2015 recommendations on accounting and analysis of the traffic accidents on the roads of the Russian Federation.

12. A. Novikov, I. Novikov, A. Katunin, A. Shevtsova, Adaptation capacity of the traffic lights control system (TSCS) as to changing parameters of traffic flows within intellectual transport systems (ITS). Transportation Research Procedia, 20, pp.455 462 (2017)

13. A. Novikov, I. Novikov, A. Katunin, A. Shevtsova, Research of influence of dynamic characteristics for options controlled intersection. Procedia Engineering, 187, pp. 664 - 671 (2017)

14. V.A. Korchagin, A.N. Novikov, S.A. Ljapin, Ju.N. Rizaeva, Complicated, complex self-advanced transport systems. The world of transport and technological machines, 2 (53), pp. 110-116 (2016)

15. P. Pavel, A.N. Novikov, O. Przhibyl, Associated systems and transport telematics. The world of transport and technological machines, 2 (49), pp. 96-102 (2015) 